\title{
El cuidado de sí mismo a través de los otros según los diálogos aporéticos de Platón ${ }^{1}$
}

\author{
Cristián Alejandro de Bravo Delorme ${ }^{2}$
}

Recibido: 18 de enero de 2019 / Aceptado: 25 de febrero de 2020

Resumen. Este artículo aborda el carácter terapéutico de la filosofía socrática según los diálogos aporéticos de Platón. Considero que la terapia de Sócrates es un cuidado de sí mismo cuyo ejercicio se lleva a cabo con los otros de manera dialéctica, por lo cual la naturaleza de sí mismo no es nunca resultado de una reflexión aislada, sino una búsqueda constante a través del diálogo. Para ello es necesario aclarar el sentido de la relación que establece el diálogo socrático, la estructura de la pregunta terapéutica y el objetivo que motiva la terapia socrática.

Palabras clave: diálogo; voluntad; consejo; terapia; sí mismo.

\section{[en] Care of the self through the others according to Plato's aporetic dialogues}

\begin{abstract}
In this paper I focus on the therapeutic nature of Socratic philosophy according to Plato's aporetic dialogues. I consider that Socrates therapy is a care of the self whose exercise is carried out with others in a dialectical way. Thus, the nature of the self is never the result of an isolated reflection, but a constant search through dialogue. It will be necessary to clarify the meaning of the relationship established by the Socratic dialogue, the structure of the therapeutic question and the objective that motivates Socratic therapy.
\end{abstract}

Keywords: dialogue; will; council; therapy; self.

Sumario: 1. Introducción; 2. El foco del cuidado filosófico de Sócrates; 3. La dimensión comunal del diálogo; 4. Intención y posibilidad de la pregunta socrática; 5 . La farmacología del cuidado dialéctico; 6. El diálogo como consejo; 7. Conclusión; 8. Referencias bibliográficas.

Cómo citar: de Bravo Delorme, C.A. (2021): "El cuidado de sí mismo a través de los otros según los diálogos aporéticos de Platón", en Revista de Filosofia 46 (1), 141-155.

1 Este trabajo se ha realizado dentro del proyecto I+D "Dinámicas del cuidado y lo inquietante. Figuras de lo inquietante en el debate fenomenológico contemporáneo y las posibilidades de una orientación filosófica. Configuración teórica y metodológica” (FFI12017/83770-P), financiado por el Ministerio de Innovación, Ciencias y Universidades del Reino de España.

2 Universidad de Sevilla debravo.cristian@gmail.com 


\section{Introducción}

Que la filosofía pueda considerarse como un ejercicio terapéutico no es una idea novedosa. Se podría decir que Sócrates es el primer analogado de la idea de "filósofo terapéuta". En efecto, él encarna la inquietud, la preocupación y el cuidado de sí mismo y de los otros de manera inexcusable. Epicuro, por su parte, atribuyó al filosofar una función terapéutica sin la cual la filosofía misma habría sido para él algo inútil. ${ }^{3}$ Musonio Rufo afirma, además, que "los que pretenden salvarse han

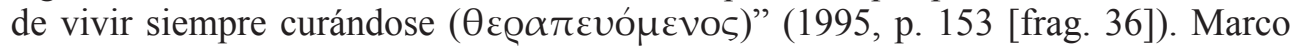
Aurelio, a su vez, puede referirse a la necesidad de un cuidado $(\theta \varepsilon \rho \alpha \pi \varepsilon i ́ \alpha)$ de la "divinidad interior" que consiste en "preservarla pura de pasión, de irreflexión y de disgusto" (1977, II, 13). Este aspecto ético de las antiguas filosofías fue consustancial a su desarrollo teorético. ${ }^{4}$ Incluso podría decirse que la ética fue la propia sustancia del filosofar antiguo siempre inmerso en su presente. Pese a que eventualmente las comunidades cristianas fueron absorbiendo este rasgo ético esencial que tenían las antiguas escuelas, la filosofía nunca olvidó su razón práctica. De ahí que Baltasar Gracián en el siglo XVII haya podido decir: "¿de qué sirve el saber si no es práctico? Y el saber vivir es hoy el verdadero saber" (2013, p. 229).

En los llamados diálogos aporéticos de Platón se puede reconocer la función terapéutica de la filosofía, aunque en un sentido distinto a las escuelas griegas y romanas que se sienten herederas igualmente de Sócrates, y diferente también, por cierto, de la psicoterapia u otras disciplinas de orientación y asesoramiento filosófico que han venido proliferando hoy en día. En los diálogos platónicos Sócrates ejerce terapéuticamente la filosofía, aunque esta terapia sólo puede comprenderse en base exclusiva a la dialéctica propia del diálogo.

Algunos comentaristas han comprendido las diversas terapias socráticas de los diálogos a partir de un enfoque evolucionista del pensamiento de Platón ${ }^{5}$. Álvaro Vallejo Campos (2016), por ejemplo, distingue tres fases en este desarrollo y argumenta que la filosofía terapéutica de Platón se desenvuelve desde el temprano y socrático diálogo Apología, donde habría una terapia enfocada exclusivamente al alma individual, pasando por el diálogo transicional Gorgias, donde la terapia sería un arte político, hasta el diálogo intermedio República, en el cual habría una terapia holística llevada a cabo por Platón con respecto al carácter utópico de la ciudad ideal (p. 227). Esto significa que la actitud de Sócrates en los diálogos habría cambiado radicalmente en relación a la verdad desde la Apología hasta la República, lo cual sería correlativo a la transformación de su carácter (el de Sócrates como portavoz de Platón) al pasar de ser un médico del alma a un médico de la cultura (p. 223).

3 "Vano es el discurso de aquel filósofo por quien no es curada ninguna afección del ser humano. Pues justamente como no asiste a la medicina ninguna utilidad si no busca eliminar las enfermedades de los cuerpos, igualmente tampoco de la filosofía si no busca expulsar la afección del alma" (1995, p. 117 [D 54]).

4 Para este enfoque terapéutico de la filosofía véase P. Hadot (1995), M. Foucault (2005), M. Nussbaum (1994). Véase también: Mace (1999), Carlisle and Ganeri (2010), Fischer (2011, pp. 49-82), Omelchenko (2012, pp. 73-81), Banicki (2014, pp. 7-31). En relación a la terapia socrática consultar Cushman (1958), Lampe (2010) y Suvák (2018). Resulta también interesante consultar a Laín Entralgo (1958) respecto al sentido de la cura por la palabra dentro del mundo griego en general. En relación a los significados de la palabra $\theta \varepsilon \varrho \alpha \pi \varepsilon i ́ \alpha$ véase Liddle and Scott (1996, pp. 792-793). En este artículo la palabra terapia será vertida como cuidado.

5 La perspectiva "evolucionista" o "genética" relativa al pensamiento de Platón proviene de Karl Friedrich Hermann (Geschichte und System der Platonischen Philosophie, Heidelberg, 1839). Para un análisis de esta perspectiva véase Tigerstedt (1974, p. 25ss). 
Por esta razón, Vallejo Campos detecta en Platón "un cambio muy importante en la concepción de la filosofía como terapia" (p. 230).

En contraste a esto, yo sugiero, en primer lugar, que lo que ocurre en los diálogos no es tanto el desarrollo intelectual del autor como el drama del personaje Sócrates. Al ser el diálogo platónico en cada caso una muestra de la interacción de Sócrates con diversos personajes y, a su vez, al ser el cuidado socrático un modo de vida superior a través del diálogo, el contenido de cada debate sólo podrá ser plenamente comprendido a la luz de la naturaleza dialéctica del pensamiento de Platón. Los interlocutores, de acuerdo al pensamiento platónico, no son meros accesorios en el debate, sino puntos de vista que poseen un sentido dramático del todo significativos, pues en éstos se revela que el contenido de cada diálogo depende de su forma. ${ }^{6}$ De ahí que cada asunto en el diálogo platónico y la forma por la cual Platón lo trata corresponda al carácter de los interlocutores que están en juego (Blondell, 2003). Por ello, si Sócrates puede llevar a cabo su terapia de distintos modos, es porque lo exige el carácter de cada interlocutor con el cual Sócrates se encuentra. En ese sentido, resulta notable la advertencia del neoplatónico Hermias. En efecto, tomando en cuenta lo que Sócrates refiere en el Fedro (271d, 277b-c), él afirma que al haber "muchas diferencias en los caracteres y prácticas de las almas, él [Sócrates] beneficia a cada uno de manera diferente, al joven de un modo, al sofista de otro modo, extendiendo sus manos a todos y exhortándolos a practicar la filosofía" $(1,1.1-5) \cdot{ }^{7} \mathrm{La}$ especial sensibilidad al texto platónico de este intérprete sugiere que la experiencia terapéutica de la filosofía es el resultado de la dinámica y de los límites del $\lambda$ ó $о \varsigma, \lambda o ́ \gamma o \varsigma$ que en cada ocasión interactúa a través de los interlocutores abriendo determinadas direcciones y vías de investigación.

Ahora bien, el cuidado de Sócrates no es terapéutico porque mediante el tratamiento dialógico el interlocutor pueda llegar a descubrir "su" verdad. La terapia de Sócrates, por de pronto, ha de explicarse con base en un diálogo por el cual ambos interlocutores pueden conjuntamente procurarse un bien (Gorgias, 505e-506a). En ese sentido, el diálogo, si es capaz de procurar un bien para ambos interlocutores, lo hará a través de las razones que el otro da y conforme a la responsabilidad del otro como base de la terapia socrática. Por lo tanto, si cada diálogo platónico puede ser interpretado de acuerdo al $\tilde{\eta} \theta$ o $\varsigma$ del interlocutor, entonces un enfoque al cuidado de Sócrates tiene que tomar en cuenta al otro con base en sus motivaciones, intenciones y deseos. Es más, si el diálogo es posible, Sócrates tiene que volver transparente lo que el interlocutor quiere. El cuidado socrático, por lo tanto, acompaña a la voluntad del otro, pues en ella se revela quién es él (Gorgias, 491e-492e). Pero quién sea él se desprenderá de lo que quiera responder (República, 340c). Dicho estrictamente, el interlocutor revelará lo que quiere al dar razón de sí mismo mediante el diálogo (Laques, 187e-188c).

En el siguiente artículo intentaré dejar en claro qué significa la terapia de

6 Este enfoque que F. Schleiermacher en los tiempos modernos puso de relieve, pero que ya resultaba operativo en muchos comentaristas antiguos, se centra en la estructura del diálogo y ha sido expresado con gran riqueza a partir del desarrollo de la fenomenología y la hermenéutica. De una manera ejemplar se puede consultar, por una parte, la interpretación de Wolfgang Wieland (1999), cuyo trabajo consiste en destacar la estructura no proposicional de los modos de conocimiento y, por otra parte, la propuesta de Stanley Rosen de una "fenomenología dramática" (1983, 1-57).

7 De ahí que Henry Teloh llame a este enfoque "el principio del Fedro" y por el cual "diferentes naturalezas psíquicas requieren diferentes logoi para su propia educación” (1986, p. 26). 
Sócrates en lo que concierne a los llamados diálogos aporéticos. Tendré que dejar aparte el importante aspecto del $\delta \alpha \mu$ óviov y la naturaleza erótica de Sócrates con el fin de enfocarme en la estructura dialéctica del cuidado socrático. ${ }^{8}$ A partir de una interpretación en clave terapéutica, será posible situar el diálogo socrático dentro de su dimensión comunal. Esto nos permitirá comprender la intención y la posibilidad de la pregunta que plantea Sócrates, la pregunta por el "es" de cierta virtud. Finalmente, podremos comprender cómo este cuidado de Sócrates permite que el interlocutor reconozca su auténtica voluntad.

\section{El foco del cuidado filosófico de Sócrates}

En la Apología, momento crucial de la vida de Sócrates, éste dice a sus conciudadanos que su única intención fue la de exhortar a cada uno a cuidar de sí mismo mediante la pregunta por la virtud (36c-38a) (Cf. De Bravo, 2019). Sócrates, planteando la pregunta por lo que sea la virtud, examina y refuta a sus interlocutores con el fin de instarlos a que sean responsables de sí mismos (29e-30a). Pero ¿qué significa este "sí mismo" que exige cuidado y en qué relación se encuentra con la virtud? Este sí mismo aparentemente sería el alma del interlocutor (Alcibiades, 130e). Sin embargo, con tal identificación el problema no queda resuelto. De hecho, este sí mismo al cual se refiere Sócrates se vuelve mucho más cuestionable si lo identificamos meramente con el alma.

Sócrates realizaba en privado ${ }^{9}$ lo que consideraba ser el más grande beneficio que podía procurarle a sus conciudadanos, a saber, intentar persuadir a cada uno "de no procurar hacer nada antes de cuidar de sí mismo lo mejor y lo más prudentemente posible, ni tampoco de procurar atender las cosas públicas de la ciudad, sino cuidar de la ciudad misma" (36c). ${ }^{10}$ Sócrates no acusa a sus conciudadanos de ser perezosos o desatentos. Lo que las palabras de Sócrates implican es que los atenienses se encontraban concernidos por cosas que impiden el cuidado de sí mismos. Los atenienses estaban concernidos por sí mismos, pero de tal modo que ellos sólo y únicamente podían hacerlo "de espaldas al fuego", para recurrir a la imagen del símil de la caverna, porque se identificaban con las opiniones de las cosas públicas. De ahí que la exhortación de Sócrates no radicara en producir un cuidado en los otros, sino, más bien, en provocar en los otros un cambio en la dirección de la mirada. Este cambio sería, así, un giro desde los asuntos en los cuales los atenienses se habían absorbido hacia sí mismos. Sócrates, sin embargo, enfatiza que esta redirección de la mirada implica suspender el cuidado de los asuntos relativos a la $\pi o ́ \lambda ı \varsigma$ y volver la mirada a la $\pi$ ó $\lambda \varsigma$ misma. Esto es muy significativo. El cuidado al que Sócrates apela no consiste en cortar la relación con los otros, ni en la separación de las cosas públicas para volverse hacia el solitario reducto de la conciencia individual. El

8 Sobre estos aspectos de la figura de Sócrates, véase Benardete (1953), Belfiore (2012) y Jedrkiewicz (2018, pp. 299-318).

$9 \quad$ El hecho de que Sócrates filosofe en privado con otros no significa que lleve a cabo conversaciones solo en espacios cerrados. La filosofía de Sócrates exige un contacto personal cercano, dentro de una casa o en el ágora. Sócrates nunca se dirige a la masa o usa procedimientos públicos para exhortar a los otros, aunque él mismo lo haga excepcionalmente a su modo en su apología (Apología, 31d5-32a3). Cf. Long (2014, pp. 8-18).

10 Todas las traducciones se basan, a veces con ligeras variantes, sobre la versión de los Diálogos (2011) de Gredos. 
cuidado socrático, a mi juicio, consiste en interrumpir el trato con las cosas de la тó $\lambda ı \varsigma$ y suspender las cosas aparentemente concernientes al individuo con el fin de volver la mirada únicamente a sí mismo, sí-mismidad que no pertenece tanto a las cosas de la ró $\lambda ı \varsigma$ como a la тó $\lambda \iota \varsigma$ misma.

Sócrates distingue, por una parte, las cosas que tienen que ver con la tó $\lambda \iota \varsigma$, a saber, el negocio, el cuidado de la propia casa, el puesto militar, la participación pública (Apología, 36b), así como los correspondientes pareceres y deseos que estas actividades generan, y, por otra parte, el sí mismo y la đó $\lambda ı \varsigma$ misma. Sócrates no comprende el sí mismo como un ente aislado e individualizado frente a una тó $\lambda ı \varsigma$ externa, sino como un modo de ser, una manera de encontrarse a sí mismo en la comunidad política. ${ }^{11}$ En ese sentido, Sócrates no sólo habría establecido una diferencia entre sí mismo y los asuntos relativos a sí mismo, sino también entre la тó $\lambda ı \varsigma$ misma y los asuntos políticos. Por lo tanto, si comprendemos este sí mismo como alma y la $\pi$ ó $\lambda \iota \varsigma$ como mundo, entonces no cabe separar ambos fenómenos como dos entes que a veces permanecen o no conectados, sino como términos correlativos y copertenecientes. Pero ¿esto significa que el alma se identificaría con su mundo? Sí mismo y $\pi 0 ́ \lambda ı \varsigma$ estarían originalmente ensamblados, aunque esta cooriginariedad no estaría exenta del problema fundamental de su unidad. ${ }^{12}$ La atención a sí mismo y a la тó $\lambda ı \varsigma$ misma, a la cual está apelando Sócrates, resulta ser un cuidado de sí mismo en conjunto. Esto significa que uno sólo se conoce a sí mismo mejor y uno sólo sabe lo que propiamente quiere a través de ejercitar un cuidado con el otro. Como intentaré mostrar este cuidado mutuo toma una forma concreta cuando los interlocutores celebran consejo entre ellos. En consecuencia, el sí mismo no parece ser algo individual, ni mucho menos es el alma como un ente encapsulado, sino, más bien, un modo de ser originariamente político. ${ }^{13}$

\section{La dimensión comunal del diálogo}

A mi juicio, lo que parece encontrarse en relación íntima con el cuidado de sí mismo

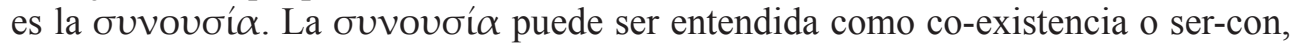

11 La $\pi o ́ \lambda ı \varsigma$ no radica en una situación geográfica ni tampoco consiste en un conjunto de procesos meramente administrativos. Aristóteles señala que la $\pi$ ó $\lambda ı \varsigma$ no tiene que ver con los límites de sus murallas (Política, 1276a25-26), por lo cual no es una localidad física, sino, más bien, un espacio de apertura donde los hombres se muestran los unos a los otros y en donde se reconocen a sí mismos como hombres a partir del ejercicio de la virtud. En la medida que cada uno tiene una función, pues el bien y la actividad radican en la función

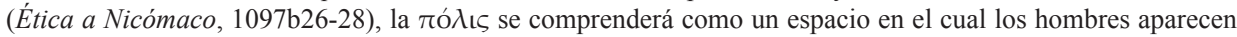
ejerciciendo sus propias funciones en relación a un bien (Política, 1252a2). Por lo tanto, el significado principal de la $\pi o ́ \lambda ı \varsigma$ reside en su carácter de ensamble y cuyo orden configura las partes de una manera orgánica.

12 Este doble enfoque, a saber, el sí mismo y la $\pi o ́ \lambda ı \varsigma$ misma, no implica que haya una identidad, sino una cierta unidad. Esta unidad, sin embargo, deviene profundamente controvertida de acuerdo a la así llamada "analogía del alma y la тó $\lambda ı \varsigma ”$ en la República y que nace en el libro II para luego ser evaluada y modificada en el libro IX (Cf. Roochnik, 2003, pp. 10-30).

13 Se podría decir que Sócrates mismo es el ejemplo del individuo, pero considero que la palabra "individuo" para retratar a Sócrates es equívoca. Sócrates es un ejemplo, pero no del individualismo, En la Apología Sócrates le dice a sus conciudadanos que el dios usa su nombre y lo pone como ejemplo como si dijera: Es el más sabio, el que, de entre vosotros, hombres, conoce, como Sócrates, que en verdad es digno de nada respecto a la sabiduría" (23b). Sócrates no es el individuo por antonomasia, sino el ejemplo de la fundamental negatividad del ser

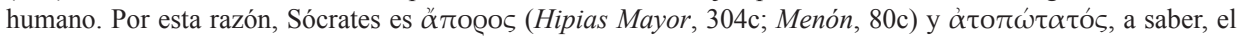
hombre más dislocado (Teeteto, 149a). 
que es el modo fundamental de ser al que Sócrates apela. Sócrates no se aleja de la tradición al invitar a co-existir, pero radicaliza en forma y contenido la unidad

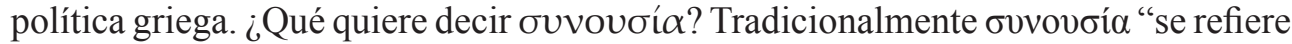
a la constante asociación de una generación más joven a hombres mayores y más cabales" (Robb, 1994, p. 197). En ese sentido, "los jovenes escuchaban, absorbían tanto la sabiduría acumulada como las habilidades de los mayores y buscaban imitar sus virtudes" (Ibidem). Esta co-existencia mediante el vínculo de la enseñanza, por lo tanto, constituye el eslabón entre las generaciones de la antigua тó $\lambda \iota \varsigma$. Con Sócrates la co-existencia toma un significado exclusivamente dialógico ${ }^{14}$. La co-

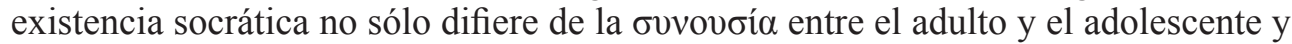

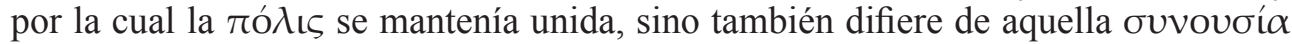
promovida por los sofistas, quienes educaban a los hombres y percibían dinero por ello (Apología, 19d-20a). El ser-con tradicional, por el cual la gimnasia y la música moldeaban el carácter, no era suficiente para Sócrates como fundamento de

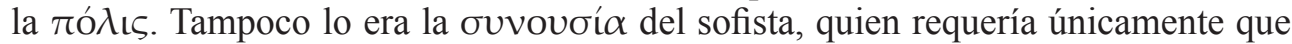
el joven estuviese atento a su largo monólogo instructivo (Protágoras, 318e-319a).

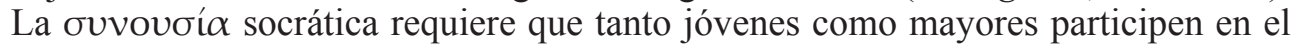
diálogo. En tanto el ser-con se encuentra basado en el diálogo, el cuidado de sí mismo deviene dialéctico. Como Bosch-Veciana lo dice con gran discreción, "para Sócrates, el pensamiento no es autosuficiente, sino que está constituido en comunión con el otro, lo cual es la verdadera ovvovóí $\alpha$, la presencia real del otro" (2004, p. 40). De acuerdo a Sócrates, sólo dialogando sobre la virtud la vida adquiere su propia virtud y claridad.

Según lo anteriomente dicho, el cuidado de Sócrates no consiste en una relación entre un experto y un estudiante. Contra lo que Terence Irwin afirma (1977, p. 75) Sócrates no cuenta con una $\tau \dot{x} \chi \nu \eta$, un conocimiento técnico por medio del cual él haga del hombre un buen ciudadano (Protágoras, 319a). Sin embargo, Sócrates es un maestro. Pero no es ese tipo de maestro que transfiere información a sus discípulos ni un maestro que es superior a los otros en términos epistemológicos (Apología, 33a-b). De acuerdo a G. A. Scott la filosofía socrática es un "interrogatorio entre dos personas, $[. .$.$] una puesta a prueba y un escrutinio frente al otro, cuya necesaria$ franqueza funciona como espejo del propio carácter" (2000, p. 154). Este ejercicio resulta posible por la conciencia de Sócrates de su ignorancia acerca del conocimiento de la virtud. ${ }^{15}$ Esta conciencia lo habilita, por una parte, a estar alerta de los límites de la sabiduría humana y, por otra parte, a escuchar el $\lambda o ́ \gamma o \varsigma$. En ese sentido Sócrates es un maestro, porque él es capaz de escuchar mejor que sus interlocutores, en la medida que el diálogo consiste fundamentalmente en obedecer el discurso, ${ }^{16}$ en ir tras la huella del argumento (Critón, 48c7; Fedro, 274a4; República, 365d2). Al escuchar el $\lambda$ ó Os Sócrates y sus interlocutores evalúan y discriminan si ellos se encuentran dirigidos a la virtud de un buen modo o de un modo deficiente durante el diálogo. ${ }^{17}$

\footnotetext{
14 Respecto al uso de ouvovoí $\alpha$ por Platón véase Tarrant (2005, pp. 133-138).

15 Este punto es muy importante, porque varios comentaristas han identificado la negación del conocimiento de la virtud por parte de Sócrates como irónica. Cf. Magrini (2018, pp. 30-38).

16 Aristóteles en la Ética a Nicómaco afirma que "tener $\lambda$ ó $\gamma$ o૬" significa en un sentido ser capaz de escuchar al

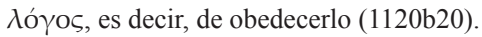

17 De acuerdo a Plutarco "la virtud sólo puede ser proporcionada por los oídos" (De Recta Ratione Audiendi, 38b).
} 
La filosofía socrática es un cuidado de sí mismo, cuya actividad se desarrolla como un diálogo entre interlocutores que son ambos buscadores y mutuamente examinadores. Sin embargo, se podría insistir en que la evidente superioridad de Sócrates es una objeción a la afirmación sobre el cuidado mutuo proporcionado por el diálogo. Pero el magisterio de Sócrates no viene de una técnica determinada y si tuviese alguna, este conocimiento no cancelaría su estado de ḋđọí $\alpha$ (Menón, 80c), pues lo que Sócrates tiene ante todo presente es precisamente que no sabe. Sócrates está siempre buscando con los otros (Cármides, 165b-c; Menón, 80d; Gorgias, 505e-506a; Protágoras, 348a-349a) y cada vez se beneficia de alguna manera de la conversación (Hipias Mayor, 304e). Sócrates co-participa y aprende conjuntamente en la persecusión de una comprensión de la virtud como un fenómeno elusivo. ${ }^{18}$ Sócrates es un buscador, el más incansable buscador entre los interlocutores, porque, como Jaspers afirma, "la esencia de la filosofía [socrática] no es la posesión de la verdad, sino la indagación de la verdad (1954, p. 12). El núcleo de la ovvovoía socrática se vuelve evidente en la dialéctica del diálogo como búsqueda conjunta de la verdadera virtud.

Por lo tanto, lo que constituye la ovvovoía socrática es la práctica del diálogo, a saber, el dar y recibir $\lambda$ ó $\gamma$ o, cuya cooperación revela un cuidado de sí mismo, incluso cuando el diálogo da ocasión al malentendido y el desacuerdo. Con todo, el diálogo de Sócrates constituye el espacio de cuidado a través de la palabra compartida. Para comprender con mayor claridad este cuidado dialéctico de la filosofía socrática, es ahora necesario explicar la intención y la posibilidad de la pregunta filosófica.

\section{Intención y posibilidad de la pregunta socrática}

El diálogo es un cuidado de sí mismo a través del cual Sócrates pregunta algo y el otro responde. Todo preguntar, como Heidegger lo ha indicado con claridad (1997, p. 30), implica: (1) lo preguntado, por ejemplo, el conocimiento o la virtud; (2) a quién se dirige la pregunta (el interrogado), acaso Teeteto o Hipócrates; (3) lo que se pone en cuestión en lo preguntado (¿qué es...?), a saber, el sentido del "es" del conocimiento o el "es" de la virtud. Y por último, pero no menos importante, (4) quien pregunta, Sócrates (el interrogador). En tanto Sócrates, consciente de no saber, plantea la correspondiente pregunta, inicia y participa de la interrogación. Al poner en cuestión el "es" de lo preguntado, Sócrates busca algo con el interlocutor y, así, presta toda su atención a quien responde. La pregunta por el "es" de lo preguntado y cuyo conocimiento es buscado, se dirige al interrogado como aquel que ya tiene un parecer sobre el bien, como aquel que ya se comporta respecto a las cosas buenas y también malas. Lo que el interlocutor cree que es verdad acerca de la moderación, por ejemplo, y lo que en cada caso él considera bueno y mejor, se encontrará en relación al modo como el interlocutor se ya encuentra dispuesto y a cómo él se mueve con las cosas que exigen moderación. En cualquier caso, ya sea que la opinión del interlocutor sobre el asunto en cuestión se encuentre de acuerdo o no con su propio

18 En Alcibiades Sócrates le dice a éste: “...debemos... perfeccionarnos. Porque lo que yo digo sobre cómo hay que educarse no es distinto para ti que para mí” (124b-c). La terapia socrática como un cuidado mutuo de sí mismo es también entendido por D. Halperin como "reciprocidad erótica" (1986, pp. 60-80). Por su parte, A. Hooper, dice: "Como amante cada uno se enfuerza hacia el bien y como amado cada uno tiene el deber de cuidar al otro y ayudarse" (2012, p. 109). 
comportamiento y padecer, lo que establece el cuidado socrático en primer lugar es que el interlocutor al responder se exhibe a sí mismo de algún modo. El interlocutor se exhibe a partir de sus creencias y deseos, con cuya identificación puede él responder a la pregunta. En correspondencia, Sócrates habla de sí mismo en una ocasión como un partero que, al interrogar por el "es" de algo, pone al descubierto al interlocutor en orden a discriminar si lo que éste da a luz es fecundo o una mera fantasmagoría (Teeteto, 149b-151b).

De acuerdo a lo anterior, se vuelve fútil plantear con respecto al diálogo la separación entre un sujeto cognoscente y un objeto independiente por conocer, en cada caso, la virtud, el conocimiento, la belleza o la piedad. El hecho de que el interrogado (Teeteto) se interese por lo preguntado o que, en cambio, la pregunta le parezca obvia e insignificante (Hipias, Eutifrón, Laques), pone de manifiesto que no existe una brecha entre el interrogado y lo preguntado. Tanto la diligencia como el descuido y la presunción dan cuenta que el interrogado y lo preguntado ya se encuentran bajo una cierta relación (Kirkland, 2012, p. 26). La transparencia de esta relación, a saber, la del que responde con aquello sobre lo cual responde, dependerá sólo y únicamente del modo como el interrogado se encuentra concernido por el "es" de lo preguntado. En ese sentido, lo que se encuentra en juego en el diálogo es cómo responde el interrogado, pues, de acuerdo a su modo de esforzarse por la verdad, se volverán transparentes para el interrogado tanto sus propios límites como su alcance cognoscitivo (Teeteto, 186a-187c).

Esto significa, entonces, que el interrogado, a través de los intentos de responder a la pregunta, de algún modo puede transparentarse a sí mismo al buscar el "es" que se encuentra puesto en cuestión. Por ejemplo, si Cármides responde que la moderación es calma (Cármides, 159b), no se debe sino a que él es capaz de estar calmado ante determinadas situaciones, como así pareciera su fama dar testimonio de ello. El "es" en cada pregunta socrática correspondería, tal como se declara en el Sofista, a la $\delta u ́ v \alpha \mu \iota \varsigma$ (247 e4), ${ }^{19}$ poder, capacidad, posibilidad, no meramente lógica, sino, discursiva y ética. El "es" que está puesto en juego en la pregunta corresponde, por lo tanto, al modo de la respuesta, cuyo contenido veritativo no depende tanto de la corrección de los enunciados del interlocutor como de la dirección de su propio comportamiento en relación a lo que responde. De ahí que pueda suceder aquella paradoja según la cual el mismo Sócrates, pese a no ser capaz de responder plenamente a las propias preguntas que plantea, pueda, sin embargo, comportase virtuosamente, tanto al dialogar como al tratar con los otros.

Si el interlocutor al responder a la pregunta de Sócrates por el "es" ya se ha identificado con los contenidos de las proposiciones que enuncia, entonces su

19 Aún cuando este artículo se encuentra enfocado en los diálogos aporéticos, esto no obsta la referencia a otros diálogos como el Teeteto y el Sofista, los cuales algunos comentaristas consideran que pertenecen a la "etapa madura" de Platón. Sin embargo, tanto el Teeteto como el Sofista son en realidad diálogos aporéticos. La conclusión del primero es claramente aporética y el carácter aporético del segundo puede ser deducido de la evidente insatisfacción de Sócrates con lo que el Extranjero de Elea ha logrado (Cf. Político, 257b2-4) Consultar el comentario de Francisco J. González a este pasaje (pp. 1997, pp. 43-46). Además, la "cronología de composición" de los diálogos y el "enfoque evolucionista" del pensamiento platónico son suposiciones que tratan de explicar contradicciones en el diálogo. Pero, en mi opinión, no hay contradicción alguna, sino problemas cuyo enfoque depende del contexto y de la persona con quien Sócrates habla (Cf. De Bravo, 2020). En tanto los diálogos no muestran la evolución del autor, sino modos diferentes de enfrentar los problemas por parte de Sócrates, no sólo es legítima la intertextualidad, sino del todo necesaria para una plena comprensión de la filosofía de Sócrates y del proyecto entero de Platón. 
respuesta de algun modo lo revelará. Dependiendo de esta identificación, el diálogo será más o menos fructífero. Teeteto, por ejemplo, al querer responder y, a su vez, al permitir ser refutado, se distingue, precisamente, por su disposición a poner en ejecución eso mismo por lo cual se le pregunta, esto es, el conocer. Al identificarse con lo que él mismo afirma y dice junto a Sócrates, Teeteto "llega a ser" su propia posibilidad de conocer, pese a que pueda errar en la respuesta. La pregunta, en ese sentido, no exige una definición proposicional que determine el "es" de lo preguntado. La pregunta exige, más bien, que el interrogado, al intentar definir el "es", se posibilite a sí mismo en la respuesta. Sin embargo, al preguntar Sócrates por el "es" y al fracasar el interlocutor al responder, éste y aquel quedan en $\alpha$ tro@í $\alpha$, lo cual no es meramente la experiencia de una mera irresolución lógica respecto a algo, sino una suerte de apertura e indecisión por la cual quienes hablan pueden evaluar, discriminar y alcanzar mayor transparencia sobre ellos mismos. Sólo así el diálogo posibilita que el interrogado se identifique con su respuesta y que se vuelva, por lo tanto, responsable de sí mismo.

De acuerdo a la intención de la pregunta y conforme a lo que la pregunta posibilita es necesario ahora despejar otro momento del cuidado dialéctico de Sócrates.

\section{La farmacología del cuidado dialéctico}

Si, como se señala en el Teeteto (189e-190a), el pensamiento es una conversación interna del alma consigo misma, entonces el diálogo no sería otra cosa que el despliegue del pensamiento. Ahora, aunque el diálogo socrático lleva a cabo un cuidado de sí mismo, no se puede pasar por alto que el $\lambda$ ó yos común y corriente tiene la tendencia a caer en el mal uso y arruinarse, por lo cual el sí mismo inmediatamente se mantiene desfigurado por medio de la $\delta o ́ \xi \alpha$, la oratoria y la erística. Por ello las posibilidades y motivaciones de los interlocutores de Sócrates resultan a veces ambiguas (Teages, Hipócrates, Critón) y, en algunos casos, se encuentran enmascaradas (Hipias, Eutifrón). Tales ejemplos dan testimonio de que el hablar con otro se vuelve para algunos una manera de adular y complacer los prejuicios de la masa (Polo) o bien una mera habilidad refutatoria (Dionisodoro y Eutidemo). El diálogo, entonces, se convierte en una discusión agonal, cuya dirección no se encuentra orientada hacia sí mismo, sino hacia la victoria de la propia posición (Eutidemo, 271c-272a). De ahí que, ante la amenaza de la imposibilidad del diálogo, sea necesario entre los interlocutores esclarecer expresamente unas determinadas condiciones para el buen desarrollo de la conversación (Cf. Vigo, 2001, pp. 5-41). De hecho, Sócrates, al advertir en una ocasión que Protágoras, en vez de preguntar y responder recíprocamente, se extendía en largos monólogos, amenaza con dejar la reunión, considerando que tal situación no satisfacía las condiciones mínimas para el diálogo. En efecto, a Sócrates sólo le interesa ponerse a prueba con el otro y poner

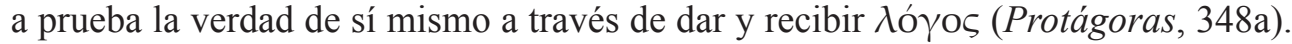

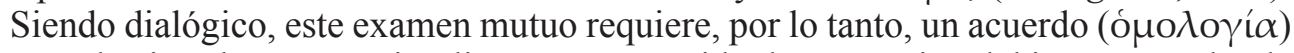
entre los interlocutores e implica, en este sentido, la aceptación del justo proceder de la conversación (Cf. Gorgias, 487e, 487e, 488a, Critón, 49d, 52d, Eutifrón, 6a, 10c, Lisis, 214d, Cármides, 162e, 172e, 175c, etc.).

El diálogo socrático supone, entonces, un justo acuerdo entre las partes acerca de lo que allí y en ese momento se encuentra en discusión. Ahora bien, si uno de los 
interlocutores, al aceptar las razones de los acuerdos, no reconoce las consecuencias de su concesión, ya sea porque se resiste a las pruebas dadas o bien porque, al verse en $\alpha \dot{\pi}$ o@í $\alpha$, considera su reputación amenazada, entonces el diálogo conlleva la posibilidad de la refutación ( $\left.{ }^{\prime} \lambda \varepsilon \gamma \chi 0 \varsigma\right),{ }^{20}$ la cual, en el caso de Sócrates, tiene el carácter de un fármaco. ${ }^{21}$ Puede entenderse la naturaleza farmacológica de la refutación de acuerdo a las palabras de Sócrates en el Gorgias (458a-b), ${ }^{22}$ donde el $\varepsilon^{\prime} \lambda \varepsilon \gamma \chi 0 \varsigma$ es usado para referirse a la liberación de una falsa opinión. Pese a la intención purificadora de Sócrates, la refutación es experimentada en la mayoría de los casos como si fuese un veneno. ${ }^{23}$ Sin embargo, este fármaco es del todo necesario, si el interrogado se encuentra de soslayo a sí mismo y dominado por sus propios pareceres.

Sócrates, al preguntar por el "es" de algo y al buscar con el otro su respuesta, exhorta al interrogado a tomar distancia de lo que tiene por verdadero. Se vuelve necesario para el interlocutor, por lo tanto, una puesta entre paréntesis de lo que en cada caso considera bueno y malo. Pero esta puesta entre paréntesis no resulta ser el primer paso del proceso terapéutico, sino, más bien, una situación que si no se mantiene suficientemente abierta durante todo el diálogo puede ocluirse o quedar atrás, como si fuese mero punto de partida. Si, en cambio, la distancia que se abre durante el diálogo deja en suspenso aquello que el interlocutor tiene por verdadero, entonces el interlocutor puede en este proceso reconocer su propio bien o, al menos, barruntarlo. En este sentido, la refutación tendrá como función "purificar" la relación del interrogado con lo que le parece bueno para sí mismo, limpiarla de adherencias injustificadas y, en suma, evaluar lo que quiere. De este modo, el sentido de la refutación dentro del cuidado dialéctico no tiene que ver con una sanción de los contenidos proposicionales de los discursos. Más bien, la naturaleza farmacológica de la refutación tiene que ver con una purificación crítica que expulsa el vicio del alma, como dice el extranjero en el Sofista (230b-e).

La refutación propia del cuidado dialéctico, según lo que ya se ha dicho, se basa en una purificación de la relación con aquello que al interlocutor le parece bueno. En este sentido, la eficacia del fármaco dependerá del modo como el interlocutor se encuentre abierto a la pregunta. Intentaré mostrar que la función purificadora del cuidado socrático es provocar en el interlocutor un movimiento deliberativo acerca de lo que él considera bueno para sí mismo. La deliberación, sin embargo, sólo resultará posible si el interlocutor quiere responder a la pregunta de Sócrates.

\section{El diálogo como consejo}

En tanto el diálogo es un cuidado de sí mismo a través del cual quien pregunta y quien responde se presentan como mutuos examinadores, el interlocutor de Sócrates se vuelve del todo relevante. El rol del interlocutor no es de ninguna manera pasivo

\footnotetext{
$20 \quad$ Para un análisis del significado del ع́̉ $\varepsilon \gamma \chi 0 \varsigma$ ver Scott (2002).

21 Derrida (1975, pp. 91-261) hace uso del significado del fármaco para considerar la crítica de los textos escritos de acuerdo al famoso pasaje del Fedro (274d-275b). El contexto dentro del cual Derrida usa la palabra, por lo tanto, es diferente al mío, aunque es posible pensar que el texto escrito, en vez de ser un veneno para el diálogo, puede ser de ayuda, si la deconstrucción derridiana es capaz de ir más allá del contenido del texto.

22 Ver también Sofista (230b-e).

23 De ahí la ambigüedad del significado de la palabra griega $\phi \alpha ́ \varrho \mu \alpha \kappa o v ~(C f$. Rinella, 2010, pp. 73.74).
} 
y, a su vez, el rol de Sócrates no radica en dictaminar lo que el interlocutor debería o no hacer. El rol de Sócrates consiste principalmente en preguntar de tal modo que el interrogado se haga responsable de lo que diga y que, por lo tanto, quiera responder. Este querer es, en este sentido, la clave para comprender la naturaleza de la pregunta terapéutica. ¿En qué sentido la voluntad del interlocutor se encuentra en juego en su respuesta?

Ante todo, es necesario advertir cómo Sócrates entiende la voluntad. En el diálogo Gorgias Sócrates ofrece una muy significativa pista. Sócrates le dice lo siguiente a su interlocutor: "Escucha, entonces, Gorgias. Debes saber que yo estoy persuadido ( $\dot{\mu} \mu \alpha v \tau o ̀ v ~ \pi \varepsilon \hat{\imath} \theta \omega$ ) de ser uno de esos que en una discusión con alguien en verdad se encuentra queriendo ( $\beta o v \lambda o ́ \mu \varepsilon v o \varsigma)$ conocer el asunto sobre el cual se debate" (453a-b). Sócrates se encuentra persuadido de ser alguien que quiere que el asunto en cuestión permanezca lo más transparente posible. Lo que la confesión de Sócrates lleva a pensar es que la voluntad se genera por la capacidad de persuadirse a sí mismo y, por así decir, de obedecerse a sí mismo. Para entender esto es necesario desentrañar ciertas relaciones lingüísticas que permitan enfocar adecuadamente el fenómeno de la voluntad. ${ }^{24}$ La palabra griega más cercana a nuestra palabra

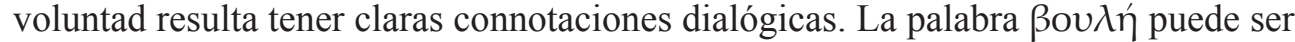
entendida como determinación y propiamente significa asamblea, consejo. Esto lleva a considerar la relación que hay entre la voz activa $\beta o v \lambda \varepsilon v ́ \omega$ (celebrar consejo) y la voz media ßoú verbos $\pi \varepsilon i ́ \theta \omega$ (persuadir) y $\pi \varepsilon i ́ \theta \omega \mu \alpha \mathrm{t}$ (ser persuadido, obedecer) ${ }^{25}$ En este sentido, parece aceptable pensar que el querer sea análogo al obedecer, si consideramos, a su vez, la persuasión en relación al consejo. Por lo tanto, no parecería errado pensar que querer algo implique la capacidad de escuchar y, en ese sentido, la disposición a obedecer el consejo que se ha tomado. Por lo tanto, no parece errado pensar que "querer" algo implique la capacidad de escuchar y, en ese sentido, la disposición a obedecer. La voluntad, de acuerdo a estas relaciones, no significaría un arbitrario y caprichoso impulso, sino la capacidad para llevar a cabo lo que cada uno se debe a sí mismo. De ahí que en este punto se revele la negatividad del ser humano, su falta esencial, por la cual la voluntad sólo se vuelve auténtica si se alcanza la conciencia del deber propio.

Si este razonamiento resulta admisible, entonces Sócrates busca precisamente que el interlocutor se encuentre dispuesto a evaluar sus propios deseos, incluso al punto de "violentarlo" (República, 514c), para que él sea persuadido por el consejo celebrado con el otro sobre su propio bien. ${ }^{26}$ Considérese el caso que ocurre cuando un médico aconseja a su paciente a tomar un determinado medicamento. El médico lo obliga a querer lo que necesita para volver a estar sano, lo persuade a hacer lo que debe para recuperarse de su enfermedad o, al menos, intenta persuadirlo. El médico puede persuadir, porque tiene autoridad, porque sabe qué es necesario para la salud

24 Albrecht Dihle (1982) afirma que hay varias palabras con diversos matices para entender el fenómeno de la

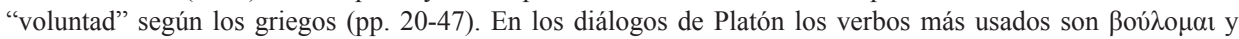

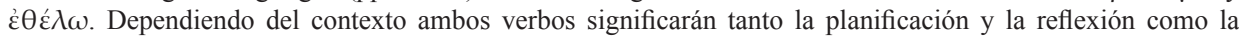
disposición y la preparación.

25 Le debo al profesor José de la Cruz Garrido la consideración de estas relaciones.

26 No resulta posible extenderme aquí en el problema de la relación entre la voluntad y el bien según los diálogos de Platón, pues para ello haría falta un análisis de la República en relación a otros diálogos. Para ello remito a Moss (2006, pp. 503-535) y Barney (2010, pp. 34-64). 
del paciente. Si, por otro lado, el médico no pudiese persuadir, no vendría de su incapacidad profesional, sino de su ineficaz retórica. Con todo, ante la autoridad del consejo médico el paciente tiene que obedecer. La diferencia entre el consejo médico y el consejo terapéutico del diálogo, sin embargo, radica en que Sócrates, en sentido estricto, no aconseja, porque no sabe. Más bien, Sócrates y su interlocutor celebran juntos consejo como consultantes. En este sentido Sócrates puede decirle a Alcibiades en una ocasión: "Pero nosotros tenemos que celebrar consejo en común

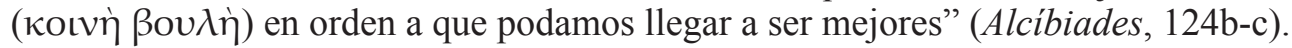

Sin embargo, en algunos casos este consejo cae en la ruina por la incapacidad

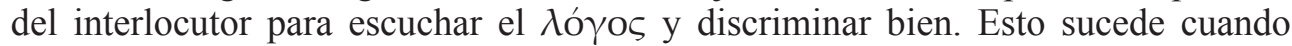
el interlocutor es llevado por su obstinación. En el Gorgias, por ejemplo, Calicles

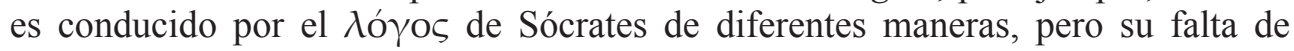
sinceridad y su miedo a la multitud que con mirada inquisitiva está presente en la discusión, lo fuerzan a aceptar a regañadientes lo argumentos de Sócrates. Al final, sin embargo, su propia disposición lo lleva a no querer responder más a las preguntas de Sócrates (519d). A su vez, quien, al escuchar la pregunta quiere responder, se encuentra dispuesto al mutuo examen de lo que cada uno tiene por verdadero. De este modo, los interlocutores pueden deliberar en conjunto sobre lo que es mejor para sí mismos. La voluntad, en este sentido, se convierte en la marca de la cooperación del diálogo y el diálogo se vuelve, así, un consejo, como si fuese una asamblea donde se decide en conjunto acerca de lo que cada uno quiere y acerca de lo que cada uno debe hacer. De este modo, si el interrogado quiere responder a la pregunta, entonces se encuentra dispuesto a sostener hasta el final la concesión de los acuerdos mutuos, incluso, en perjuicio de sus propias creencias y opiniones. Obligado, así, por la pregunta, el interrogado sería capaz de persuadirse a través de la refutación de sus anteriores convicciones y podría, asimismo, volverse obediente al consejo deliberado en conjunto acerca de sí mismo.

Dentro del diálogo terapéutico el querer exige un componente deliberativo que le aporta un carácter obligante a la voluntad. Sin embargo, cuando un interlocutor se encuentra auto-engañado y de este engaño se alimenta su voluntad, el fármaco socrático funciona como una fuerza que distancia al interlocutor de sus propias creencias. En la mayoría de los casos los interlocutores responden las preguntas de Sócrates y, en principio, se someten a la terapia socrática, pero ellos no pueden hacerse cargo del todo de las consecuencias de sus propias respuestas. Por lo tanto, ellos no están dispuestos a hacerse responsables de sus respuestas y a celebrar el consejo necesario sobre lo que es mejor para sí mismos. Sin embargo, como R. Cushman dice, el propósito de Socrates sería "despertar del sueño las verdaderas opiniones que cada interrogado ha fingido negar pero en las que realmente cree" (1958, p. 308).

\section{Conclusión}

El fracaso del cuidado dialéctico de Sócrates y, por lo tanto, la frustración del consejo, no proviene tanto de una impotencia de la dialéctica como de la incapacidad de escuchar del interlocutor. Al preguntar Sócrates busca que el interlocutor quiera responder y lo ayuda a que se vuelva responsable de su propia respuesta (Alcibiades, 127e). Lo que Sócrates quiere, en último término, es abrir la posibilidad de la 
autotransparencia a través de la cooperación dialógica. Esta autotransparencia, sin embargo, no implica la posesión del bien, sino el propio diálogo y el mutuo examen de sí mismo.

En términos generales cabe decir que el cuidado socrático está hecho de la misma materia que la enseñanza socrática. Dicho más precisamente, el cuidado socrático constituye el carácter mismo de la enseñanza de los diálogos platónicos. En efecto, basta considerar el proceso que se lleva a cabo entre el esclavo de Menón y Sócrates (Menón, 82b-85b) para advertir que la educación consiste ante todo en la destrucción o disolución crítica de aquellos pasos dados, casi inercialmente, por mor de la reconstrucción de lo planteado, en el caso del Menón, el doble del cuadrado. Esta destrucción de las creencias, de los pareceres inerciales para aprender dialécticamente algo es análoga al proceso según el cual el interlocutor se cuida a sí mismo mediante el cuidado (farmacológico) del otro. Tal como uno no puede cuidarse a sí mismo sin el otro, así resulta necesario el otro para aprender por uno mismo. Bajo la auténtica cooperación dialógica de los interlocutores Sócrates puede realizar su especial terapia, esto es, el ejercicio político fundamental por medio del cual la voluntad delibera a través del mutuo examen qué es lo mejor para sí mismo (Alcibiades, 124e).

\section{Referencias bibliográficas}

Aristóteles (1999): Ética a Nicómaco. Edición bilingüe y traducción de María Araujo y Julián Marías. Madrid, Centro de estudios políticos y constitucionales.

Aristóteles (1959): Politics. Translated by H. Rackman. Cambridge MA: Harvard University Press.

Aristóteles (1988): Política. Traducción de Manuela García Valdés. Madrid: Gredos

Banicki, K. (2014): "Philosophy as Therapy: Towards a conceptual model", Philosophical Papers, 43:1, pp. 7-31.

Barney, R. (2010): "Plato on the Desire for the Good". In: S. Tenenbaum (ed.). Desire, practical reason, and the good. Oxford, Oxford University Press, pp. 34-64.

Belfiore, E. (2012): Socrates'Daimonic Art. Love for Wisdom in Four Platonic Dialogues. Cambridge, Cambridge University Press.

Benardete, S. (1953): The Daimonion of Socrates. An Interpretative Study of Plato's Theages. Chicago, Illinois, The University of Chicago.

Blondell, R. (2003). The Play of Characters in Plato's Dialogues. Cambridge, Cambridge University Press.

Bosch-Veciana, A. (2004): "Els diàlegs socràtics de Plató com a escenificació d'una synousia i el seu valor filosòfic", İtaca. Quaderns Catalans de Cultura Clàssics. 20, pp. 33-61.

Bueno, G. (1954): "La esencia del teatro". Revista de ideas estéticas. Abril, Mayo, Junio, no 46 , pp. $15-39$.

Bueno, G. (1982): "Psicoanalistas y epicureos. Ensayo de introducción del concepto antropológico de "heterías soteriológicas". El Basilisco, n¹3, 1981-82, pp. 12-39.

Burnet, J. (1900-1907): Platonis Opera. 5 volumes. Oxford, E. typographeo Clarendoniano.

Carlisle, C. \& Ganeri, J. (2010) (ed.): Philosophy as Therapy, Cambridge, Cambridge University Press.

Carey, J. (2019): “Socrates' Exhortation to Follow the Logos". In: P. Diduch \& M. Harding (eds.). Socrates in the Cave. On the Philosopher's Motive in Plato. Switzerland, Palgrave Macmillan. 
Couvreur, P. (1901): Hermiae Alexandrini in Platonis Phaedrus Scholia. Paris, Librairie Émile Bouillon, Editeur.

Cushman, R. (1958): Therapeia: Platon's Conception of Philosophy. Chapell Hill, The University of North Carolina Press.

De Bravo, C. (2020): "Puntos de vista de la verdad: sobre el carácter polifónico del pensamiento platónico". Kriterion, vol.61, n.145, pp. 131-149.

De Bravo, C. (2019): "Límite de la téchne y virtud del diálogo según Platón". Trans/Form/ Ação, Marília, v. 42, n. 4, pp. 9-28.

Derrida, J. (1975): Diseminación. Traducido por J. M. Arancibia. Madrid, Editorial Fundamentos.

Dihle, A. (1982): The Theory of Will in Classical Antiquity, California, University of California Press.

Epicteto, Musonio, Rufo (1995): Tabla de Cebes, Disertaciones, Fragmentos menores, Manual, Fragmentos. Traducción de Paloma Ortíz García. Madrid, Gredos.

Epicuro (1995): Obras Completas. Traducción de J. Vara. Madrid, Cátedra.

Fischer, E. (2011): "How to practice philosophy as therapy: Philosophical therapy and therapeutic philosophy", Metaphilosophy, Vol. 42, Nos. 1-2, January 2011, pp. 49-82.

Foucault, M. (2005): The Hermeneutics of the Subject. Translated by G. Burchell. New York: Palgrave MacMillan.

González, F. (1997): “On the way to Sophia: Heidegger on Plato’s Dialectic, Ethics, and Sophist". Research in Phenomenology 27 (1), pp. 16-60.

Gracián, B. (2013): Oráculo manual y arte de prudencia. Edición de Emilio Blanco. Madrid, Cátedra.

Hadot, P. (1995): Philosophy as a way of life. Spiritual exercises from Socrates to Foucault. Translated by M. Chase. Oxford, Blackwell Publishers.

Halperin, D. (1986): "Plato and Erotic Reciprocity", Classical Antiquity, Vol. 5, No. 1, pp. 60-80.

Heidegger, M. (1997): Ser y Tiempo. Traducción, prólogo y notas de J. E. Rivera. Santiago de Chile, Editorial Universitaria.

Hooper, A. (2012): "The Dual-Role Philosophers: An Exploration of a Failed Relationship". In: M. Johnson and H. Tarrant (eds.): Alcibiades and the Socratic Lover-Educator, London, Bristol Classic press.

Irwin, T. (1977): Plato's Moral Theory. The Early and Middle Dialogues. Oxford, Clarendon Press.

Jaspers, K. (1954): The way to wisdom. Translated by R. Manheim. New Haven, Yale University Press.

Jedrkiewicz, S. (2018): “A Literary Challenge: How to Represent Socrates’ Daimonion”. In: Socrates and the Socratic Dialogue, Leiden, Boston, Brill, pp. 299-318.

Kirkland, S. (2012): The Ontology of Socratic Questioning in Plato's Early Dialogues. Albany, New York, State University of New York.

Lampe, K. (2010): "Socratic Therapy from Aeschines of Sphettus to Lacan", Classical Antiquity, Vol. 29, No. 2 (October 2010), pp. 181-221.

Liddle, H. G. and Scott, R. (1996): A Greek-English Lexicon. Oxford, Clarendon Press.

Long, C. (2014): Socratic and Platonic Political Philosophy, Cambridge, Cambridge University Press.

Mace, C. (1999): Heart and Soul. The therapeutic face of philosophy, London, Routledge.

Marco Aurelio (1977): Meditaciones. Traducción de R. Bach Pellicer. Madrid, Gredos. 
Moss, J. (2006): "Pleasure and illusion in Plato", Philosophy and Phenomenological Research $72(3)$, pp. 503-535.

Nussbaum, M. (1994): The Therapy of Desire. New Jersey, Princeton University Press.

Omelchenko, N. (2012): "Philosophy as Therapy", Diogenes, 57, (4), pp. 73-81.

Plato (2005): Euthyphro, Apology, Crito, Phaedo, Phaedrus. Translated by Harold North Fowler. Cambridge, Harvard University Press.

Platón (2011): Diálogos I y II. Varios traductores. Madrid: Gredos.

Plutarch (1927): Moralia, vol. I. Translated by F. C. Babbitt. Cambridge MA, Harvard University Press.

Rinella, M. (2010): Pharmakon. Lanham, Lexington Books.

Robb, K. (1994): Literacy and Paideia in Ancient Greece. Oxford, Oxford University Press.

Roochnik, D. (2003): Beautiful City. The Dialectical Character of Plato's Republic. Ithaca and London, Cornell University Press.

Rosen, S. (1983): Plato's Sophist. The Drama of Original and Image. New Haven and London, Yale University Press.

Suvak, V. (2018): "Socratic Therapy as Taking Care of the Self and Others", en: Suvák, V. (ed.): Care of the Self. Ancient Problematizations of Life and Contemporary Thought. Leiden, Boston, Brill.

Tarrant, H. (2005): "Socratic Synousia: A Post-Platonic Myth?", Journal of the History of Philosophy, Volume 43, Number 2, April, pp. 131-155.

Teloh, H. (1986): “The Importance of Interlocutors' Characters in Plato's Early Dialogues”, Proceedings of the Boston Area Colloquium in Ancient Philosophy, 2, Brill, pp. 25-38.

Tigerstedt, E. N. (1974): Interpreting Plato. Stolckholm, Sweden, Almqvist \& Wiksell International.

Vallejo Campos, A. (2016): Socrates as a physician of the soul in Gabriele Cornelli (ed.), Plato's Styles and Characters. Between Literature and Philosophy. Berlin, Boston, De Gruyter.

Vigo, A. (2001): Platón, en torno a las condiciones y la función del diálogo cooperativo. Tópicos, número 008-009, Santa Fé, Argentina, pp. 5-41.

Wieland, W. (1999): Platon und die Formen des Wissens. Göttingen, Vandenhoeck und Ruprecht. 